\title{
Turbulent driving scales in molecular clouds
}

\author{
C. M. Brunt ${ }^{1}$, M. H. Heyer ${ }^{2}$, and M.-M. Mac Low ${ }^{3}$ \\ 1 Astrophysics Group, School of Physics, University of Exeter, Stocker Road, Exeter, EX4 4QL, UK \\ e-mail: brunt@astro.ex.ac.uk \\ 2 Department of Astronomy, University of Massachusetts at Amherst, 710 North Pleasant Street, Amherst, MA 01003, USA \\ e-mail: heyer@astro.umass.edu \\ 3 Department of Astrophysics, American Museum of Natural History, 79th Street and Central Park West, New York, \\ NY 10024-5192, USA \\ e-mail: mordecai@amnh.org
}

Received 5 February 2009 / Accepted 22 July 2009

\section{ABSTRACT}

\begin{abstract}
Context. Supersonic turbulence in molecular clouds is a dominant agent that strongly affects the clouds' evolution and star formation activity. Turbulence may be initiated and maintained by a number of processes, acting at a wide range of physical scales. By examining the dynamical state of molecular clouds, it is possible to assess the primary candidates for how the turbulent energy is injected.

Aims. The aim of this paper is to constrain the scales at which turbulence is driven in the molecular interstellar medium, by comparing simulated molecular spectral line observations of numerical magnetohydrodynamic models and molecular spectral line observations of real molecular clouds.

Methods. We use principal component analysis, applied to both models and observational data, to extract a quantitative measure of the driving scale of turbulence.

Results. We find that only models driven at large scales (comparable to, or exceeding, the size of the cloud) are consistent with observations. This result applies also to clouds with little or no internal star formation activity.

Conclusions. Astrophysical processes acting on large scales, including supernova-driven turbulence, magneto-rotational instability, or spiral shock forcing, are viable candidates for the generation and maintenance of molecular cloud turbulence. Small-scale driving by sources internal to molecular clouds, such as outflows, can be important on small scales, but cannot replicate the observed large-scale velocity fluctuations in the molecular interstellar medium.
\end{abstract}

Key words. magnetohydrodynamics (MHD) - turbulence - techniques: spectroscopic - ISM: molecules - kinematics and dynamics - radio lines: ISM

\section{Introduction}

Turbulence is an important agent that controls the evolution (and perhaps formation) of molecular clouds and the subsequent production of stars. As such, it has attracted significant attention from theorists, especially since the advent of numerical supercomputer simulations. Of particular interest is the source(s) of energy injection that create and sustain turbulence in molecular clouds. A number of different mechanisms have been proposed, including supernovae, HII regions, outflows, spiral arms, magneto-rotational instability in galactic disks (Miesch \& Bally 1994; Mac Low \& Klessen 2004). These mechanisms may be distinguished by the effective spatial scale at which they preferentially operate, and clues to the nature of the energy injection mechanism(s) may be extracted from spectral line imaging observations of molecular clouds.

A number of methods for studying resolved velocity fields in molecular clouds have been developed and applied. These include projected velocity (line centroid) analysis (e.g. Scalo 1984; Miesch \& Bally 1994; Ossenkopf \& Mac Low 2002; Brunt \& Mac Low 2004), the spectral correlation function (SCF; Rosolowsky et al. 1999), velocity channel analysis (VCA; Lazarian \& Pogosyan 2000, 2004), and principal component analysis (PCA; Heyer \& Schloerb 1997). To date, these methods have been used to estimate the power law indices of the velocity structure function/power spectrum in molecular clouds from observed data cubes of molecular line emission (e.g. Brunt \& Heyer 2002b; Heyer \& Brunt 2004).

Application of PCA to Outer Galaxy molecular clouds (Brunt 2003a - Paper I hereafter) revealed that, in comparison to simple models, the observational record favored large-scale driving of turbulence in the molecular clouds. In their study of the Polaris molecular cloud, Ossenkopf \& Mac Low (2002) also found that large-scale driving of turbulence provided a better explanation of the cloud's velocity structure.

In this paper, we construct simulated observations of molecular clouds, derived from computational simulations of interstellar turbulence. The models include magnetic fields and selfgravity and are driven (randomly forced) on a range of spatial scales. We employ PCA to quantitatively investigate the observational signatures of different driving scales. Our numerical measurements are compared to previous PCA results obtained from the simple cloud models of Paper I and to the same measurements made on real molecular clouds. The layout of the paper is as follows. In Sect. 2, we briefly summarize the PCA method and review the relevant findings of Paper I. Section 3 introduces the numerical models and summarizes the simulated observations of these. In Sect. 4, we present our results, compare these to corresponding observations, and discuss the implications for the generation of turbulence in molecular clouds. Our conclusions are given in Sect. 5 . 


\section{PCA}

PCA can be used to decompose three dimensional spectral line imaging observations onto orthogonal spectroscopic eigenvectors along which ordered sources of variance in the data are maximized (Heyer \& Schloerb 1997). Projection of the data onto the eigenvectors produces a sequence of diagnostic eigenimages. We refer below to each coupled eigenvectoreigenimage pair as a principal component (PC), distinguished by its order $m=1,2, . ., N$, where $N$ is the number of spectroscopic channels of the data set. The amount of variance in the data accounted for by the PCs is a decreasing function of $m$. The characteristic sizes of eigenimage structures are measured as the spatial scale at which their autocorrelation function (ACF) falls to $1 / e$ of the zero-lag value (Brunt \& Heyer 2002a). At order $m$ we denote the characteristic spatial scale of the eigenimage as $l_{m}$.

In the literature there are numerous examples of eigenimages obtained from simple molecular cloud models and from observations of real molecular clouds (e.g. Heyer \& Schloerb 1997; Brunt 1999; Brunt 2002b). Real molecular cloud eigenimage sequences display chaotic structures that are only replicated by models that contain chaotic (turbulent) velocity fluctuations on all scales. A quantitative statement on this was given in Paper I, as summarized below.

The analysis of Paper I considered fractional Brownian motion velocity fields with correlated velocity fluctuations up to a maximum size scale defined by the turnover wavenumber, $k_{\text {cut }}$, in the velocity power spectra; $k_{\text {cut }}$ determines the largest wavelength, $\lambda_{0}$, at which correlated velocity fluctuations are present. For wavenumbers greater than $k_{\text {cut }}$, the power spectrum was a power law, while for wavenumbers less than $k_{\text {cut }}$ the power spectrum was flat. For the simple models of Paper $\mathrm{I}, \lambda_{0}$ is used as a surrogate for the driving scale, $\lambda_{\mathrm{D}}$. The model velocity fields of Paper I were then embedded in a "cloud" - this was simply a Gaussian density distribution parameterized by the spatial FWHM, $L_{\mathrm{c}}$. The combined density and velocity fields were then transferred to the observational axes via a density-weighted projection of the line-of-sight velocity field.

Upon applying PCA, it was found that the ratio of characteristic spatial scales, $l_{2} / l_{1}$, derived from the first two eigenimages, was sensitive to variations in $\lambda_{0} / L_{\mathrm{c}}$. In detail: $l_{2} / l_{1}$ was tightly correlated with $\lambda_{0} / L_{\mathrm{c}}$ for $\lambda_{0} / L_{\mathrm{c}}<1$. For models with $\lambda_{0} / L_{\mathrm{c}}>1$, no correlation of $l_{2} / l_{1}$ with $\lambda_{0} / L_{\mathrm{c}}$ was observed, but all models with $\lambda_{0} / L_{\mathrm{c}}>1$ could be readily distinguished from models with $\lambda_{0} / L_{\mathrm{c}}<1$.

When these models were compared to spectral line observations of real molecular clouds, it was found that only models which included large-scale velocity fluctuations could match the observational data. We now repeat the analysis of Paper I using more realistic molecular cloud models obtained via numerical simulation of driven turbulence. Radiative transfer of ${ }^{12} \mathrm{CO}$ and ${ }^{13} \mathrm{CO}(J=1-0)$ spectral lines was included in the construction of the "observable" models. Both of these features are an advance over Paper I.

\section{Numerical data}

\subsection{Overview}

We use simulations of randomly driven hydrodynamical (HD) turbulence and magnetohydrodynamical (MHD) turbulence (Mac Low 1999), performed with the astrophysical MHD code
ZEUS-3D ${ }^{1}$ (Clarke 1994), a 3D version of the code described by Stone \& Norman (1992a,b). Further details on the numerical scheme are found in Mac Low (1999). To drive the turbulence, a fixed pattern of Gaussian fluctuations is drawn from a field with power only in a narrow band of wavenumbers around some value $k_{\mathrm{d}}$. The dimensionless wavenumber(s) $k_{\mathrm{d}}$, at which the simulations are driven, counts the number of driving wavelengths $\lambda_{\mathrm{d}}$ in the computational box. This pattern is normalized to produce a set of perturbations that are added to the velocity field, with the amplitude chosen to maintain constant kinetic energy input rate. This offers a very simple approximation to driving by mechanisms that act on a particular scale. In general, one must recognize the possibility of multi-scale energy injection from a variety of sources (Scalo 1987). However, for our purposes here, the numerical simulations provide a conveniently parameterized sample of "clouds" with which to investigate the observational signatures of different driving scales. We also include models with self-gravity in which the turbulence is driven at small and large scales (Klessen et al. 2000). In these models, turbulence is initiated in the fluid and allowed to reach steady state before self-gravity is turned on. We include snapshots of these models at a number of timesteps $\left(t / t_{\mathrm{ff}}=0,1,5 / 3\right)$ where $t_{\mathrm{ff}}$ is the free-fall timescale and $t=0$ refers to the point at which self-gravity is turned on.

A summary of the models is given in Table 1. The models are scale free; we impose physical units as follows: mean density $n_{\mathrm{H}_{2}}=139 \mathrm{~cm}^{-3}$; linear size $L=10 \mathrm{pc}$; sound speed $c_{s}=0.265 \mathrm{~km} \mathrm{~s}^{-1}\left(T_{k}=17 \mathrm{~K}\right.$; isothermal $)$ - see Mac Low (1999), Klessen et al. (2000). All simulations were performed on a $128^{3}$ grid.

\subsection{Simulated observations}

To generate observed simulations directly comparable to real data, we apply radiative transfer calculations to the numerically simulated velocity and density fields The physical fields are transferred onto the observational axes using a non-LTE excitation calculation that accounts for local radiative trapping at each grid point, followed by radiative transfer through the grid (see Brunt \& Heyer 2002a). The intensities of the ${ }^{13} \mathrm{CO}$ and ${ }^{12} \mathrm{CO}$ spectral lines are computed at velocity resolution $0.05 \mathrm{~km} \mathrm{~s}^{-1}$.

The "cloud size" for the simulations is, nominally, the size of the computational box. However, the simulated density fields (particularly for small-scale driving) do not have sufficient (column) density contrast to enable a meaningful measurement of $l_{1}$ because the ACF of the first eigenimage does not fall to the $1 / e$ point to which the spatial scale measurements are referenced. This could be avoided by padding the fields before ACF computation, but this is a poor choice as the fields are actually periodic. In order to ensure a more meaningful "cloud size" for the models, we have defined a spherical window of 100 pixels diameter within the computational box. Within this window, the density field is taken as simulated, and we taper to zero density quickly but smoothly outside this window. We take the "cloud size" as the diameter of the imposed spherical window, denoted as $L_{\mathrm{c}}$.

The driving wavelength, $\lambda_{\mathrm{D}}$ is $N_{\text {pix }} /(\min ) \lambda_{\mathrm{d}}$ where $N_{\text {pix }}=128$ and $(\min ) \lambda_{\mathrm{d}}$ is the smallest wavenumber within the driving range (i.e. 1,3 , or 7 ; see Table 1 ). This results in values of $\lambda_{\mathrm{D}}=128,42.7$, and 18.3, and values of the "fractional driving scale" $\lambda_{\mathrm{D}} / L_{\mathrm{c}}=1.28,0.427$, and 0.183 .

\footnotetext{
1 Available from the Laboratory for Computational Astrophysics at http://lca.ucsd.edu/portal/software/zeus-3d
} 
Table 1. Numerical models: parameters and PCA measurements.

\begin{tabular}{lcccccc}
\hline \hline Model & $k_{\mathrm{d}}{ }^{a}$ & $M^{b}$ & $v_{A} / c_{s}{ }^{c}$ & $\lambda_{\mathrm{D}} / L_{\mathrm{c}}$ & $l_{2} / l_{1}\left({ }^{13} \mathrm{CO}\right)$ & $l_{2} / l_{1}\left({ }^{12} \mathrm{CO}\right)$ \\
\hline HA8 & $7-8$ & 1.9 & 0 & 0.18 & $0.07 \pm 0.01$ & $0.05 \pm 0.01$ \\
HC8 & $7-8$ & 4.1 & 0 & 0.18 & $0.06 \pm 0.01$ & $0.06 \pm 0.01$ \\
HE8 & $7-8$ & 8.7 & 0 & 0.18 & $0.03 \pm 0.01$ & $0.04 \pm 0.01$ \\
HC4 & $3-4$ & 5.3 & 0 & 0.43 & $0.12 \pm 0.02$ & $0.12 \pm 0.02$ \\
HE4 & $3-4$ & 12.0 & 0 & 0.43 & $0.31 \pm 0.04$ & $0.20 \pm 0.02$ \\
HC2 & $1-2$ & 7.4 & 0 & 1.28 & $0.25 \pm 0.03$ & $0.30 \pm 0.03$ \\
HE2 & $1-2$ & 15.0 & 0 & 1.28 & $0.26 \pm 0.03$ & $0.31 \pm 0.03$ \\
MC81 $v_{\|}$ & $7-8$ & 3.5 & 1 & 0.18 & $0.05 \pm 0.01$ & $0.05 \pm 0.01$ \\
MC81 $v_{\perp}$ & $7-8$ & 3.5 & 1 & 0.18 & $0.05 \pm 0.01$ & $0.04 \pm 0.01$ \\
MC85 $v_{\|}$ & $7-8$ & 3.4 & 5 & 0.18 & $0.04 \pm 0.01$ & $0.03 \pm 0.01$ \\
MC85 $v_{\perp}$ & $7-8$ & 3.4 & 5 & 0.18 & $0.04 \pm 0.01$ & $0.04 \pm 0.01$ \\
MC41 $v_{\|}$ & $3-4$ & 4.7 & 1 & 0.43 & $0.10 \pm 0.02$ & $0.14 \pm 0.02$ \\
MC41 $v_{\perp}$ & $3-4$ & 4.7 & 1 & 0.43 & $0.10 \pm 0.02$ & $0.10 \pm 0.02$ \\
MC45 $v_{\|}$ & $3-4$ & 4.8 & 5 & 0.43 & $0.14 \pm 0.02$ & $0.11 \pm 0.02$ \\
MC45 $v_{\perp}$ & $3-4$ & 4.8 & 5 & 0.43 & $0.12 \pm 0.02$ & $0.10 \pm 0.02$ \\
MC4X $v_{\|}$ & $3-4$ & 5.3 & 10 & 0.43 & $0.12 \pm 0.02$ & $0.12 \pm 0.02$ \\
MC4X $v_{\perp}$ & $3-4$ & 5.3 & 10 & 0.43 & $0.11 \pm 0.02$ & $0.12 \pm 0.02$ \\
ME21 $v_{\|}$ & $1-2$ & 14.0 & 1 & 1.28 & $0.55 \pm 0.06$ & $0.75 \pm 0.08$ \\
ME21 $v_{\perp}$ & $1-2$ & 14.0 & 1 & 1.28 & $0.72 \pm 0.09$ & $0.48 \pm 0.05$ \\
D1H $\left(t / t_{\mathrm{ff}}=0\right)$ & $1-2$ & 10.0 & 0 & 1.28 & $0.62 \pm 0.03$ & $0.43 \pm 0.04$ \\
D1H $\left(t / t_{\mathrm{ff}}=1\right)$ & $1-2$ & 10.0 & 0 & 1.28 & $0.13 \pm 0.03$ & $0.29 \pm 0.04$ \\
D1H $\left(t / t_{\mathrm{ff}}=5 / 3\right)$ & $1-2$ & 10.0 & 0 & 1.28 & $0.28 \pm 0.06$ & $0.22 \pm 0.02$ \\
D3H $\left(t / t_{\mathrm{ff}}=0\right)$ & $7-8$ & 10.0 & 0 & 0.18 & $0.04 \pm 0.01$ & $0.03 \pm 0.01$ \\
D3H $\left(t / t_{\mathrm{ff}}=1\right)$ & $7-8$ & 10.0 & 0 & 0.18 & $0.04 \pm 0.01$ & $0.04 \pm 0.01$ \\
$\mathrm{D} 3 \mathrm{H}\left(t / t_{\mathrm{ff}}=5 / 3\right)$ & $7-8$ & 10.0 & 0 & 0.18 & $0.04 \pm 0.01$ & $0.04 \pm 0.01$ \\
\hline
\end{tabular}

${ }^{a}$ Driving wavenumber.

${ }^{b}$ rms Mach number.

${ }^{c}$ Ratio of Alfvén speed to sound speed.

\section{Turbulent driving scales}

\subsection{Results}

PCA was applied to the simulated observations according to the procedure given in Brunt \& Heyer (2002a). The characteristic spatial scales, $l_{1}$ and $l_{2}$, are derived from the first two eigenimages of each simulation and the ratios, $l_{2} / l_{1}$, are listed in Table 1 . Figure 1 shows these measurements plotted against $\lambda_{\mathrm{D}} / L_{\mathrm{c}}$ and compared to the simple cloud results of Paper I. The $l_{2} / l_{1}$ measurements from the numerical models are in good agreement with the simple fBm cloud results of Paper I. Figure 1 verifies that $l_{2} / l_{1}$ provides a coarse measure of the turbulent driving scale. Note that for $\lambda_{\mathrm{D}} / L_{\mathrm{c}}>1\left(l_{2} / l_{1}>0.1-0.2\right)$ there is little or no sensitivity to the actual driving scale, and this regime should be viewed simply as "large scale driving". According to the results of Paper I, the variation of $l_{2} / l_{1}$ between $\sim 0.2$ and 0.8 occurs naturally, due to the unpredictability of the projection of a largescale velocity gradient onto the line of sight. The results presented here show that the magnetic model driven at large scales, ME21, has a larger $l_{2} / l_{1}$ than the hydrodynamic models HC2, HE2. In light of the Paper I results, not too much should be read in to this result without further study. Similarly, temporal variations in $l_{2} / l_{1}$ for the D1H model should not be over-interpreted.

A visual example of the data presented in Table 1 is given in Fig. 2, where we display the first four eigenimages obtained from ${ }^{12} \mathrm{CO}$ simulated observations of HE2, HE4, and HE8 (c.f. Fig. 3 of Paper I). Figure 2 also includes the first four eigenimages obtained from ${ }^{12} \mathrm{CO}$ observations of the NGC 7538 molecular cloud, for which $l_{2} / l_{1}=0.26 \pm 0.09$. Figure 2 demonstrates that for turbulence driven on small scales, the higher order $(m>1)$ eigenimage structures are confined to small scales relative to the overall cloud size. Cloud models with large-scale driving of turbulence generate large second eigenimage structures with respect to the overall cloud size, typically displaying a positivenegative "dipole" structure. The ratio $l_{2} / l_{1}$ is a simple quantitative measure of this trend.

It is evident from Fig. 1 that there is a small trend for the recovered $l_{2} / l_{1}$ to be very slightly larger than the results found for the fBm fields of Paper I (this is most evident in panels (b) and (d) of Fig. 1). Inspection of the power spectra of the model velocity fields reveals the likely origin of this effect. In Paper I, the fBm velocity fields were designed to have a power law spectrum at wavenumbers greater than a cut-off wavenumber, $k_{\text {cut }}$; above this wavenumber, the power was flat (independent of $k$ ). The numerically simulated velocity fields, on the other hand, have excess power relative to the fBm models at low wavenumbers. A representative example of this is demonstrated in Fig. 3 where the model $\mathrm{HC} 8$, driven at $k_{\mathrm{d}}=7-8$ is compared to an "equivalent" fBm model with $k_{\text {cut }}=7$. While an obvious turnover in spectral power is clearly evident at $k<k_{\mathrm{d}}$ for $\mathrm{HC} 8$, it is not as sharp as the corresponding $\mathrm{fBm}$ field that used as a surrogate in Paper I. Figure 1 demonstrates, however, that the driving scale is still recoverable using PCA.

Another important consideration is the effect of radiative transfer of the spectral lines. To investigate this, densityweighted velocity histograms (e.g. Falgarone et al. 1994) were also constructed as an approximation to a perfectly excited optically thin spectral line observation (referred to below as " $v$-hist" models). The $v$-hist models provide a baseline for investigating the effect of saturation on the analysis. As the $v$-hist models include no saturation effects, we used these to examine any biases arising from the use of ${ }^{13} \mathrm{CO}$ and ${ }^{12} \mathrm{CO}$ where opacity and excitation effects are present. Figure 4 a compares $l_{2} / l_{1}$ derived from ${ }^{13} \mathrm{CO}$ and ${ }^{12} \mathrm{CO}$ with $l_{2} / l_{1}$ derived from the $v$-hist method. At 


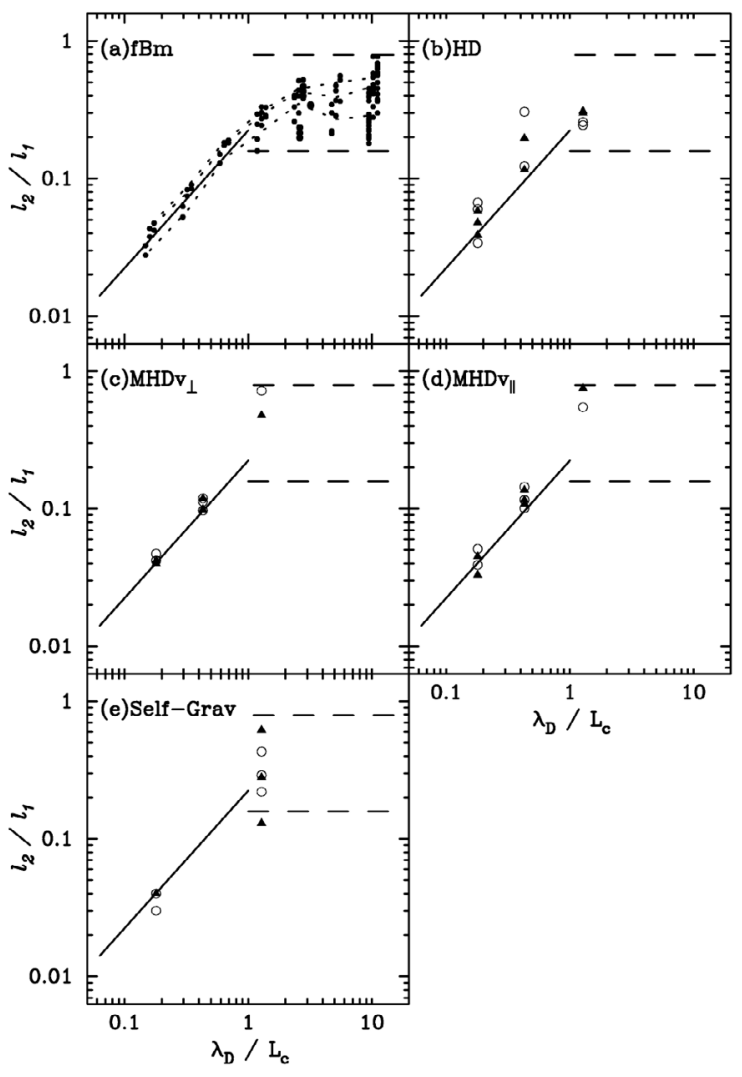

Fig. 1. a) Plot of the ratio of scales from the second and first eigenimages, $l_{2} / l_{1}$, versus the driving scale ratio $\lambda_{\mathrm{D}} / L_{\mathrm{c}}$ (dots) obtained from PCA of simple model clouds (Paper I). The solid line marks the trend of $l_{2} / l_{1}$ with $\lambda_{\mathrm{D}} / L_{\mathrm{c}}$ for $\lambda_{\mathrm{D}} / L_{\mathrm{c}}<1$. The dashed lines mark the range of $l_{2} / l_{1}$ observed when $\lambda_{\mathrm{D}} / L_{\mathrm{c}}>1$. b) Plot of $l_{2} / l_{1}$ versus $\lambda_{\mathrm{D}} / L_{\mathrm{c}}$ for the HD simulations. c) Plot of $l_{2} / l_{1}$ versus $\lambda_{\mathrm{D}} / L_{\mathrm{c}}$ for MHD $\left(v_{\perp}\right)$. d) Plot of $l_{2} / l_{1}$ versus $\lambda_{\mathrm{D}} / L_{\mathrm{c}}$ for MHD $\left(v_{\|}\right)$. e) Plot of $l_{2} / l_{1}$ versus $\lambda_{\mathrm{D}} / L_{\mathrm{c}}$ for the selfgravitating models. In panels (b)-(e) ${ }^{12} \mathrm{CO}$ and ${ }^{13} \mathrm{CO}$ measurements are represented by open circles and triangles, respectively.

small $l_{2} / l_{1}$ (i.e. small $\lambda_{\mathrm{D}} / L_{\mathrm{c}}$ ) there are no systematic effects arising from opacity in the spectral lines. However, at higher $l_{2} / l_{1}$ the $\mathrm{CO}$ emission overestimates $l_{2} / l_{1}$ relative to $v$-hist. This effect starts to become evident at $l_{2} / l_{1} \approx 0.1-0.2$, which, as shown in Fig. 1 , is the point at which $\lambda_{\mathrm{D}} / L_{\mathrm{c}} \approx 1$ (i.e. the turbulence is driven at the scale of the cloud). For $l_{2} / l_{1}$ (or $\left.\lambda_{\mathrm{D}} / L_{\mathrm{c}}\right)$ greater than this transition point, $l_{2} / l_{1}$ not surprisingly loses any sensitivity to the actual driving scale. We conclude that there are no serious problems arising from opacity effects, and simply note that values of $l_{2} / l_{1}$ greater than $\sim 0.1-0.2$ are indicative of large-scale driving of turbulence. Interestingly, values of $l_{2} / l_{1}$ derived from real molecular clouds can significantly exceed 0.2 (this is not typically seen in $v$-hist models), and we identify the source of this as opacity effects in clouds driven at large scales. In Fig. 4b we compare $l_{2} / l_{1}$ derived from ${ }^{13} \mathrm{CO}$ and ${ }^{12} \mathrm{CO}$. While noting the difference between the $\mathrm{CO}$ observations and $v$-hist observations, there is clearly no systematic difference found between ${ }^{13} \mathrm{CO}$ and ${ }^{12} \mathrm{CO}$. This is in accord with previous investigations of PCA for other applications (Brunt 2003b). Finally, we note that the inclusion of self-gravity does not have any effect on the observed $l_{2} / l_{1}$, as can be seen in Fig. 1 .

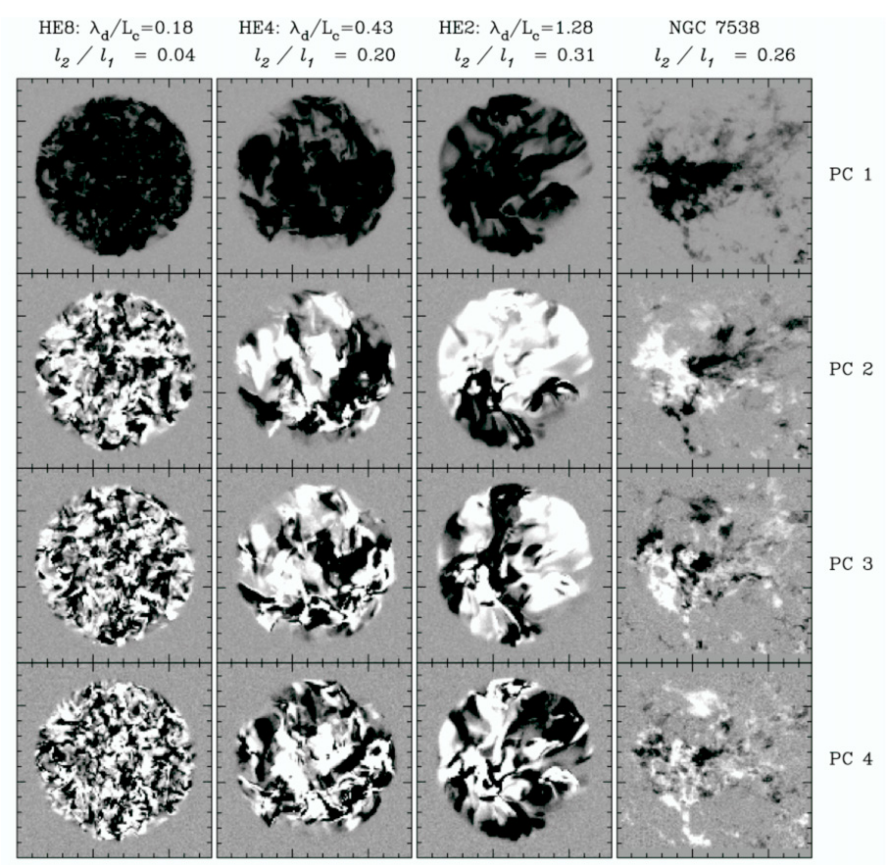

Fig. 2. Example ${ }^{12} \mathrm{CO}$ eigenimage sequences for the first four principal components obtained from the HD data for $\lambda_{\mathrm{D}} / L_{\mathrm{c}}=0.18,0.43$, and 1.28 and from observations of the NGC 7538 molecular cloud. The eigenimages of molecular clouds are more similar to the numerical cloud models with turbulence driven at scales comparable to the cloud size.

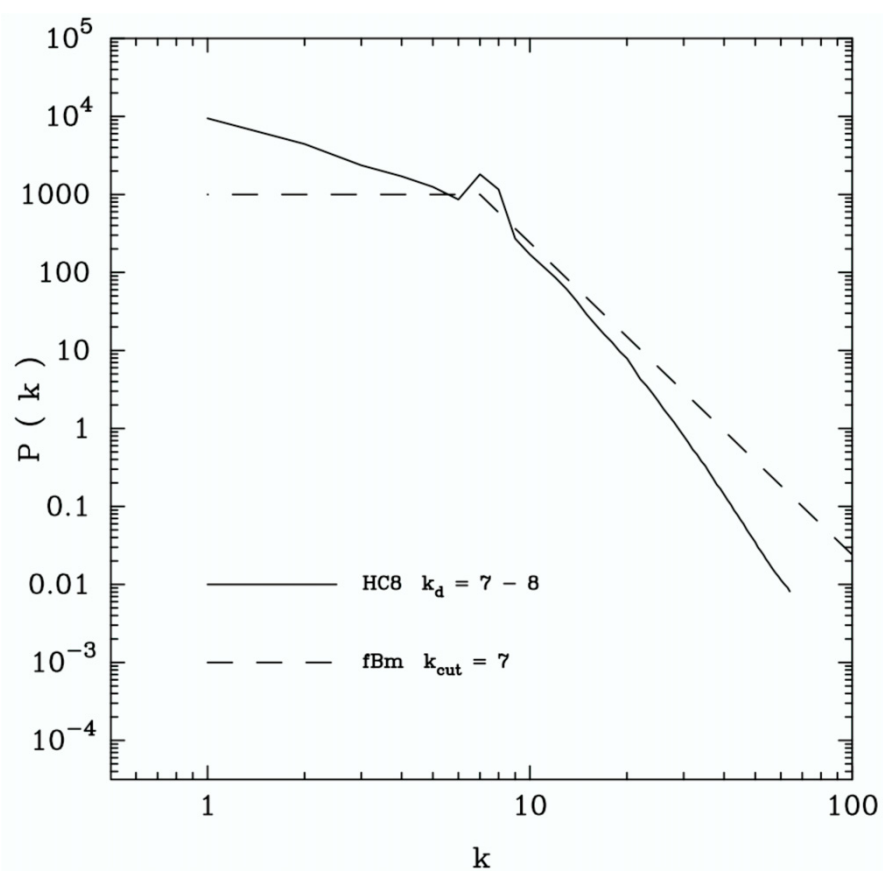

Fig. 3. Comparison of velocity power spectra (power $P$ versus wavenumber $k$ ) obtained from a numerically simulated cloud (HC8, with $\left.k_{\mathrm{d}}=7-8\right)$ and an $\mathrm{fBm}$ field $\left(k_{\text {cut }}=7\right)$ of the type used in Paper I to represent turbulent driving at $k \approx 7$. HC8 has more power at low wavenumbers relative to the $\mathrm{fBm}$ field. (The vertical scale in this plot is arbitrary.)

\subsection{Discussion}

Both analytical and computational descriptions of turbulence are necessarily constrained by observations of interstellar clouds. A qualitative inspection of Fig. 2 shows that the eigenimages 

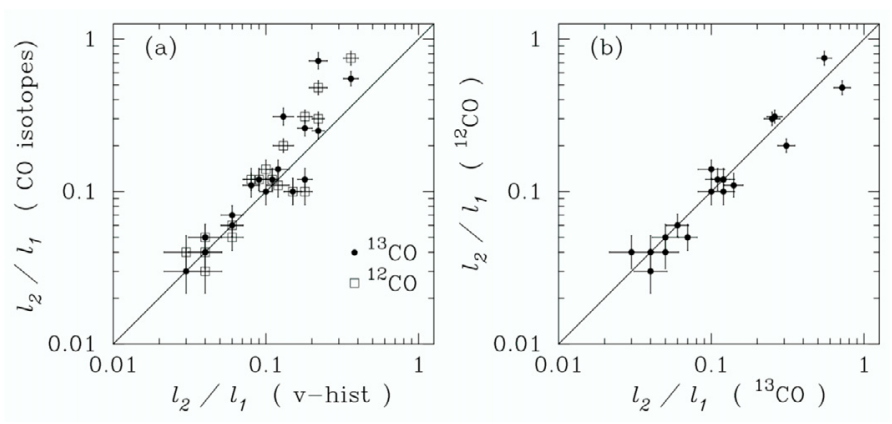

Fig. 4. a) Comparison of $l_{2} / l_{1}$ derived from simulated $\mathrm{CO}$ observations and observations using the $v$-hist method where opacity and excitation effects are not included. b) Comparison of $l_{2} / l_{1}$ derived from simulated ${ }^{13} \mathrm{CO}$ and ${ }^{12} \mathrm{CO}$ observations. For each plot, the solid line denotes equivalent values along the ordinate and absissca axes.

derived from clouds models with large $\lambda_{\mathrm{D}} / L_{\mathrm{c}}$ are more consistent with the observations of NGC 7538. More generally, the measured values of $l_{2} / l_{1}$ from real molecular clouds are typically $\gtrsim 0.2$. In Fig. 5 we plot the histogram of $l_{2} / l_{1}$ measured in the sample of clouds from Paper I, to which we have added additional measurements from the clouds analyzed in Heyer \& Brunt (2004). In the combined sample there are 35 clouds in total. Using Fig. 1 as a guide to the relationship between $\left\langle l_{2} / l_{1}\right\rangle$ and $\lambda_{\mathrm{D}} / L_{\mathrm{c}}$, these values imply that the molecular clouds are dominated by turbulence driven on large scales compared to the cloud sizes. This may be simply a result of the driving scale itself determining the size of molecular clouds (Ballesteros-Paredes \& Mac Low 2002; Paper I).

In our experiment, we have considered the simplified case where a single "driving scale" is in operation. Within this limitation we identify large-scale driving as the dominant scenario. In reality, turbulence can in principle be driven on multiple scales by a number of mechanisms (Scalo 1987). The origin of large-scale energy injection is discussed by Mac Low \& Klessen (2004), who concluded that field supernovae were the dominant mechanism in regions where they occur, while magnetorotational instability (Kim et al. 2003; Tamburro et al. 2009) may provide a background level. In addition to these, other possible mechanisms include forcing by shocks in spiral arm potentials; Dobbs \& Bonnell (2007) demonstrate that the scale-dependent velocity dispersion in molecular clouds can be replicated by simulated clouds in a galactic disk with a fixed spiral arm pattern. Most of these processes likely require that the molecular cloud turbulence is inherited from still larger scale motions in the atomic ISM (Elmegreen 1993, Ballesteros-Paredes et al. 1999; Brunt 2003a). In this scenario, the "driving" of molecular cloud turbulence could simply be due to the continuous downward cascade of turbulent energy, that not only injects the turbulence but is also responsible for the (potentially rapid) molecular cloud formation in the first place (Bergin et al. 2004; Glover \& Mac Low 2007). The presence of large-scale turbulence in molecular clouds would be a natural, inevitable consequence of their formation, and their subsequent evolution can be significantly affected by dynamical events occurring in the larger scale ISM.

Energy injection on (initially) small scales by the spatiotemporally intermittent development of outflows, stellar winds and HII regions within the cloud may not be well modeled by random forcing methods used in these simulations. These point-like injections of energy can expand their spheres of influence over time and may ultimately contribute to large-scale turbulent motions. However, on large scales, these processes are

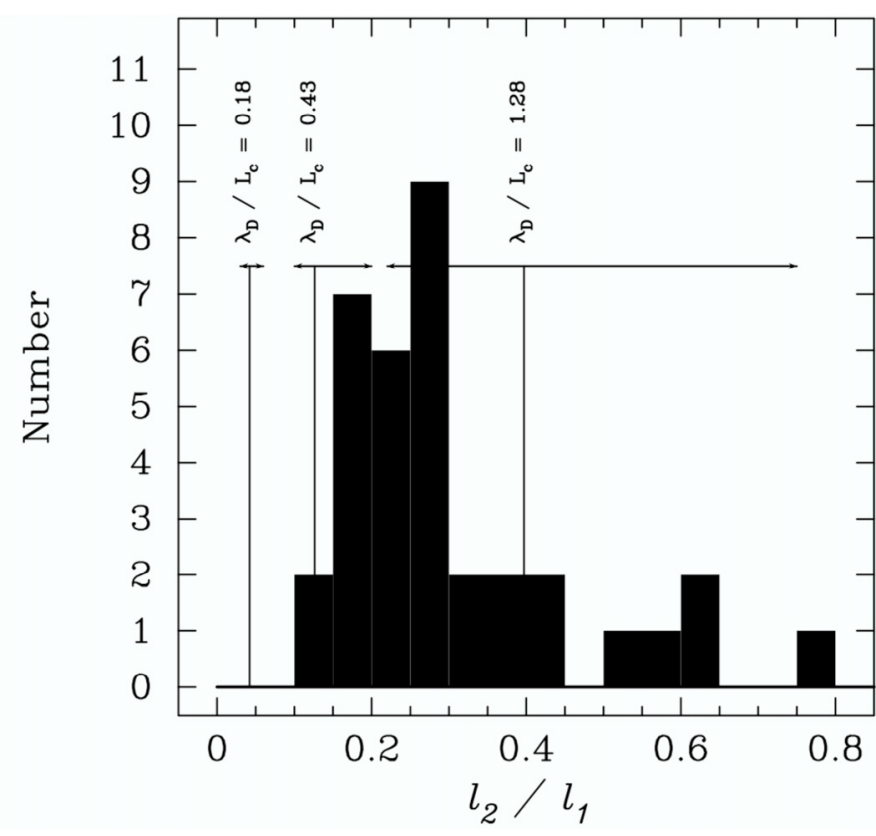

Fig. 5. Histogram of $l_{2} / l_{1}$ obtained from ${ }^{12} \mathrm{CO}$ observations of real molecular clouds. The vertical lines mark the mean $l_{2} / l_{1}$ derived from the model observations $\left({ }^{12} \mathrm{CO}\right)$ and the horizontal arrows extend over the range of measured $l_{2} / l_{1}$.

disfavored on energetic grounds (Mac Low \& Klessen 2004). While there is evidence that energy injection by outflows can be important over limited scales (e.g. Bally et al. 1996; Knee \& Sandell 2000) it is unlikely that outflow-driven turbulence can explain the origin of molecular cloud turbulence as a whole (Walawender et al. 2005; Banerjee et al. 2007). This is demonstrated by recent simulations of outflow-driven turbulence which reveal that energy injection by outflows is not capable of creating turbulence at scales comparable to the cloud size. Models of interacting outflows generated either randomly (Carroll et al. 2008), or self-consistently (Nakamura \& Li 2007) show that turbulence is only injected with an effective driving scale of about $1 / 5$ to $1 / 10$ the size of the cloud $\left(\lambda_{\mathrm{D}} / L_{\mathrm{c}} \approx 0.1-0.2\right)$ which is incompatible with our results as summarized in Figs. 1 and 5. The observable ratio $l_{2} / l_{1}$ is expected to lie in the range $0.02-$ 0.05 when $\lambda_{\mathrm{D}} / L_{\mathrm{c}} \approx 0.1-0.2$, according to our modeling results. Additionally, the cloud modeled by Nakamura \& Li (2007) is only $1.5 \mathrm{pc}$ in size, and it is unclear whether the effective driving scale would increase (for the same outflow parameterization) if a larger cloud was modeled. If the fractional driving scale of $0.1-0.2$ is interpreted as a physical driving scale of $0.15-0.3 \mathrm{pc}$, then outflow-driven turbulence would be even less effective in globally exciting turbulence in larger clouds. On the other hand, in larger clouds, more massive and energetic outflows may be expected to be present, but it is not currently clear how (or if) the effective fractional driving scale would increase.

An observational estimate of the effective driving scale of turbulence by outflows was found by Swift \& Welch (2008). They inferred an energy injection scale of 0.05 pc for L1551, which is a small fraction of the the overall cloud diameter of around $1.8 \mathrm{pc}$. Using this estimate, they found a rough balance between the energy injection rate (from the outflows) and the turbulent dissipation rate, with a characteristic injection/decay time scale of $\sim 0.1 \mathrm{Myr}$, which is substantially less than the inferred cloud age of $\sim 4-6$ Myr. We note here that some caution is required in interpreting the appearance of injection/decay balance for the outflow-driven turbulence. The dissipation time scale of 

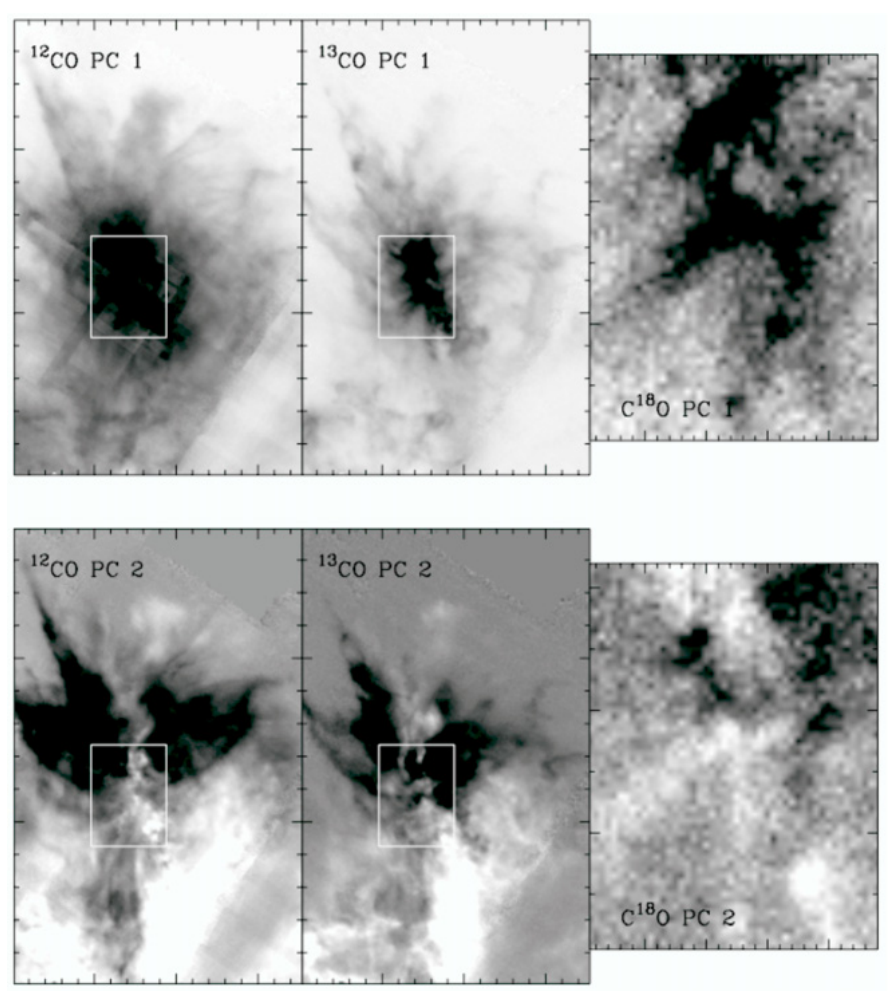

Fig. 6. First and second eigenmages obtained from PCA of the NGC 1333 molecular cloud. The $\mathrm{C}^{18} \mathrm{O}$ data and analysis are confined to the central core region, delineated by the rectangular box on the ${ }^{12} \mathrm{CO}$ and ${ }^{13} \mathrm{CO}$ images.

turbulence is proportional to the driving scale (Mac Low 1999). Swift \& Welch (2008), in calculating their dissipation rate, used a driving scale of $0.05 \mathrm{pc}$, and therefore their result shows primarily that the energy injection through outflows is quickly dissipated on short time scales over short length scales. If the L1551 cloud is, or has been, subject to large-scale $(\gtrsim 1.8 \mathrm{pc})$ driving of turbulence, then the large-scale turbulence is controlled by a much longer dissipation timescale of $(1.8 / 0.05) \times 0.1 \mathrm{Myr} \approx 3.6 \mathrm{Myr}$, which is more in line with the cloud age. The small-scale driving of outflows would then occur within the longer time evolution of the cloud, set by the longer dissipation time scales of the initial turbulence, injected on large scales.

The effect of multiple outflows within a small region of space may be seen in the outflow-rich NGC 1333 molecular cloud. Here, Quillen et al. (2005) describe as many 22 cavities within a $1 \mathrm{pc}^{3}$ volume, possibly excavated by outflow activity, in the ${ }^{13} \mathrm{CO}(J=1-0)$ map of Ridge et al. (2003). The cavities have typical diameters of $0.1-0.2 \mathrm{pc}$, indicative again of a small effective driving scale, as the shells surrounding the cavities would presumably collide and merge at larger scales. However, it is not yet clear that outflows are the driving source for these cavities, as many do not have obvious stellar sources inside them - see Quillen et al. (2005) for further discussion.

To investigate outflow-driven turbulence from an observational perspective, we applied the PCA method to CO observations of the NGC 1333 molecular cloud. We used the $J=1-0$ spectral lines of ${ }^{12} \mathrm{CO}$ and ${ }^{13} \mathrm{CO}$ observed at FCRAO as part of the COMPLETE project (Ridge et al. 2006), as well as FCRAO $\mathrm{C}^{18} \mathrm{O} J=1-0$ spectral line data toward the central core region of NGC 1333. In Fig. 6 we show the first two eigenimages obtained from the analysis for each spectral line. For ${ }^{12} \mathrm{CO}$ and ${ }^{13} \mathrm{CO}$, we find "dipole" second eigenimage structure characteristic of large-scale turbulence, and measure $l_{2} / l_{1}$ values of $0.59\left({ }^{12} \mathrm{CO}\right)$ and $0.63\left({ }^{13} \mathrm{CO}\right)$. The overall cloud size is estimated from the ${ }^{12} \mathrm{CO} l_{1}$ measurement to be $3.27 \mathrm{pc}$, assuming a distance of $318 \mathrm{pc}$. These measurements show that turbulence is (or has been) driven on large-scales in NGC 1333, and is unlikely to have originated from the outflows, which are confined to the central core region, marked by the small box in Fig. 6. Analysis of the $\mathrm{C}^{18} \mathrm{O}$ data in this box allows us to focus in on the high column density material lying in the immediate vicinity of the outflows. We measure $l_{2} / l_{1}=0.18 \pm 0.07$ for the high column density material traced by $\mathrm{C}^{18} \mathrm{O}$, which is substantially smaller than the global $l_{2} / l_{1}$ values found using ${ }^{12} \mathrm{CO}$ and ${ }^{13} \mathrm{CO}$, but still reasonably consistent with turbulence driven at large scales. Some caution should be applied to this result, because, as noted above, large temporal variations in $l_{2} / l_{1}$ can occur in the case of large scale driving. With this proviso, according to our model results, the measured $l_{2} / l_{1}$ for the central region would imply a fractional driving scale of $\lambda_{\mathrm{D}} / L_{\mathrm{c}} \approx 0.5-1.0$, or a physical driving scale of $0.43-0.86 \mathrm{pc}$, based on the measured $l_{1}=0.86 \mathrm{pc}$ for the $\mathrm{C}^{18} \mathrm{O}$ data. For reference, the cavity sizes of $0.1-0.2$ pc in NGC 1333, if taken as a measure of the driving scale within the $0.86 \mathrm{pc} \mathrm{C}^{18} \mathrm{O}$ central core region, best match our models driven at $k_{\mathrm{d}}=3-4$, for which we find $l_{2} / l_{1} \approx 0.11$. Examination of the $\mathrm{C}^{18} \mathrm{O}$ second eigenimage structure reveals that it shares, to some degree, the same north-south "dipole" structure seen in the ${ }^{12} \mathrm{CO}$ and ${ }^{13} \mathrm{CO}$ second eigenimages. The presence of this signature, along with the $l_{2} / l_{1}=0.18$ measurement, suggests that both the large-scale turbulence in the cloud as a whole, and small-scale (outflow) driven turbulence are important in this region. The inferred driving scale is therefore likely an intermediate value between that arising from the outflows and that deriving from the large-scale turbulent gradient across the core region. As a caveat, we note that the ${ }^{12} \mathrm{CO}$ and ${ }^{13} \mathrm{CO}$ lines are likely to better trace lower density, more spatially extended material than that traced by the $\mathrm{C}^{18} \mathrm{O}$ line, so the relationship between the gradients seen in Fig. 6 may not be as obvious as we assume. If the $\mathrm{C}^{18} \mathrm{O}$ gradient is itself caused by outflow activity, then this may indicate an interesting connection between the large- and small-scale energy injection mechanisms.

To examine the overall scale-dependence of turbulent motions in NGC 1333, in Fig. 7 we show plots of $\delta v$ versus $l$ for each isotope from their respective maps (see Brunt \& Heyer 2002b). It is noteworthy that the ${ }^{12} \mathrm{CO}$ and ${ }^{13} \mathrm{CO}$ measurements conform to the typical $\delta v-l$ relationship found by Heyer \& Brunt (2004). Of more interest here is the increase in $\delta v$ seen on scales of $\sim 0.1 \mathrm{pc}$ in the $\mathrm{C}^{18} \mathrm{O}$ data, relative to the overall level set by the ${ }^{12} \mathrm{CO}$ and ${ }^{13} \mathrm{CO}$ data for the cloud as a whole. This excess kinetic energy likely derives from the effect of outflows in the central core region of the cloud. Although the number of retrieved $\delta v-l$ pairs is small, the data are in broad agreement with Quillen et al. (2005) who estimate outflow-driven cavity sizes and velocity perturbations of $\sim 0.1-0.2 \mathrm{pc}$ and $\sim 1 \mathrm{kms}^{-1}$, respectively. Thus internal driving of turbulence can be important in sub-parsec regions of larger clouds, where a large number of outflows can develop. The PCA results for the NGC 1333 cloud as a whole set this in context, revealing the presence of larger-scale turbulence that will evolve on longer time scales than that present in the central core region. Turbulent dissipation in the dense part can therefore be replenished not only by local sources, but by external "driving" by larger-scale flows originating in the surrounding cloud, as part of the overall hierarchy of turbulent motions. One cannot then consider the central star-forming regions as closed systems, evolving independently of their larger scale surroundings. Our cloud sample as a whole does not support a picture in which large-scale 

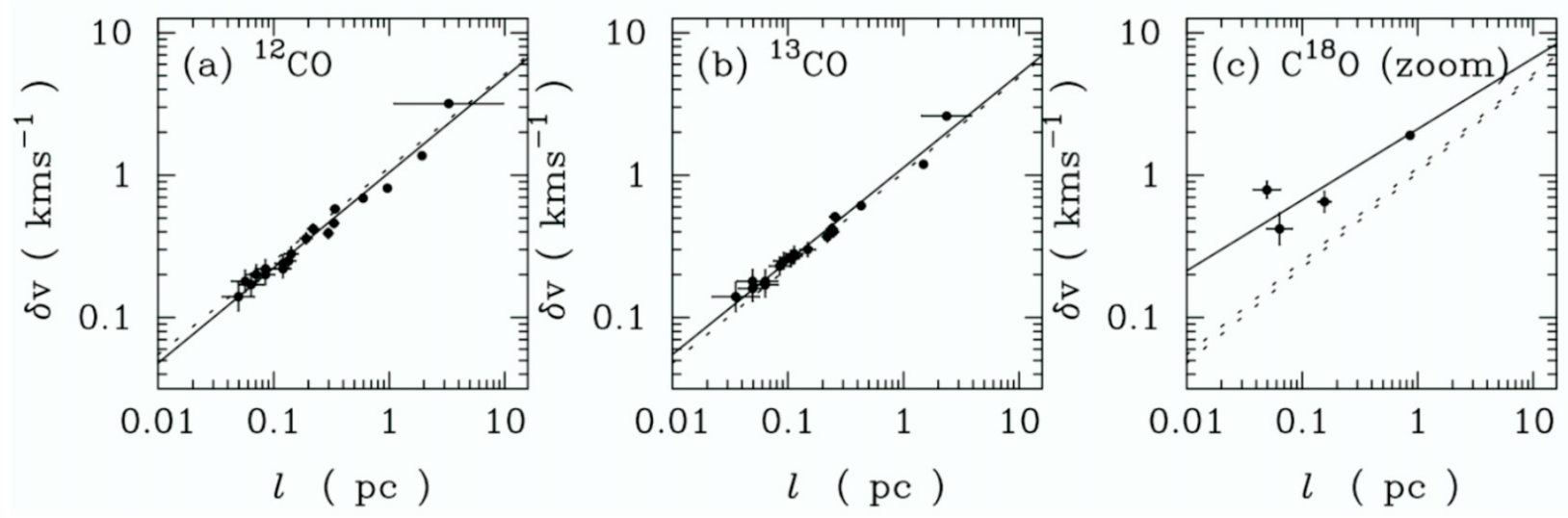

Fig. 7. Plots of $\delta v$ versus $l$ using all space and velocity scales obtained from PCA of ${ }^{12} \mathrm{CO},{ }^{13} \mathrm{CO}$, and $\mathrm{C}^{18} \mathrm{O} J=1-0$ data in the NGC $1333 \mathrm{moleclar}$ cloud. The solid lines in each plot show the bisector fit to all points; the fitted relationships for ${ }^{12} \mathrm{CO}$ and ${ }^{13} \mathrm{CO}$ are repeated as dotted lines in all plots.

turbulent motions have decayed sufficiently so that small-scale driving alone is dominant. We conclude that either the clouds are continually driven on large scales, or that most clouds are sufficiently young that the initial seeding of turbulence by the largescale flows that created the cloud has not yet dissipated. Other observations (Ossenkopf \& Mac Low 2002; Brunt \& Mac Low 2004) support this conclusion. It is not yet clear whether clouds are continually driven, or whether the turbulence is in a decaying state. Offner et al. (2008) find that while their simulated clouds do not readily distinguish between decaying or driven conditions, there is a marginal preference for continual driving. If clouds are driven at large scales, the turbulent dissipation time is comparable to their dynamical time (Mac Low 1999).

As noted above, the dipole pattern in the second eigenimage that is observed in all molecular clouds provides an important constraint to candidate driving sources. The dipole reflects the spatial distribution of the largest velocity differences within a cloud. One cannot directly discriminate whether these velocity differences are due to shear, compressive, or expanding motions. Large-scale driving can readily account for such a pattern as it directly deposits the energy at these scales. Turbulence driven on small scales can in principle provide support on larger scales (Klessen et al. 2000) and it may be possible for excess small-scale energy input to drive large scale expansion motion. More generally, a cluster-forming clump could experience expansion, collapse, or perhaps oscillation about an equilibrium state, depending on how active the star formation is. However, the dipole pattern suggests a more directed flow of material, which would require the combined action of outflows to act in a preferred direction. There is a possible mechanism for outflows to be oriented in a particular direction: a strong magnetic field could result in core collapse along field lines, leading to co-oriented protostellar disks and therefore co-oriented outflows, for which some evidence is presented in Anathpindika \& Whitworth (2008). The magnetic field strength needed to impose such directivity is likely to inhibit cluster formation, and instead promote star formation in a more distributed, quiescent mode (Heitsch et al. 2001; Price \& Bate 2008). Outflows from newborn stars and HII regions can also redistribute energy from small to larger scales by driving expanding shells. Such flows may also perturb the magnetic field that threads the molecular cloud to excite Alfvén waves that can further redistribute the outflow energy. However, such activity would again require implausible coherence of location and alignment of outflows to reproduce the observed dipole pattern.
Another candidate for driving large-scale turbulence "internally" is energy injection by HII regions, as argued by Matzner (2002). However, large-scale driving is applicable to molecular clouds where HII regions are absent, such as G216-2.5 (Maddelena's Cloud; Heyer et al. 2006). So while these mechanisms are no doubt present in some molecular clouds, they cannot explain molecular cloud turbulence in general and their effects will be limited to small scales. If HII regions become large enough to drive large-scale motions, then it is likely that the cloud will be destroyed through photoionization rather than “driven" (Matzner 2002; Dale et al. 2005).

Turbulence driven at large scales promotes star formation that is clustered, rapid, and efficient, while small-scale driving tends to form stars singly, slowly, and inefficiently (Klessen et al. 2000). If the star formation rate can be retarded by (additional) small-scale energy injection, it must do this in an environment which can be significantly (perhaps dominantly) influenced by large-scale turbulent flows of material. While the large scale versus small-scale driving picture can be modified by the effects of magnetic fields (Nakamura \& Li 2008; Price \& Bate 2008), it is in a much more dynamic way than that described by the quasistatic model (Shu et al. 1987). For example, recent high spatial dynamic range imaging of the Taurus molecular cloud (Goldsmith et al. 2008; Heyer et al. 2008) reveal large scale, magnetically regulated, turbulent flows of material.

In addition to energy injection, another important consideration is the dissipation of turbulence. Basu \& Murali (2001) argue that it is difficult to reconcile the inferred heating rate arising from dissipation of turbulence with observed cloud luminosities unless the driving occurs at large scales. More recently, Pan \& Padoan (2009) show that (assuming large-scale driving) heating by turbulent dissipation can exceed cosmic ray heating, and typical temperatures of $\sim 8.5 \mathrm{~K}$ can be sustained by turbulent heating alone. Since the turbulent heating rate scales as $\left(\lambda_{\mathrm{D}} / L_{\mathrm{c}}\right)^{-1}$, widespread small-scale driving could lead to high cloud temperatures that are incompatible with observations for molecular clouds as a whole (although not for small sub-regions within the clouds).

We mention a note of caution regarding the results presented here. The numerical simulations of turbulence relied on random forcing (in Fourier space) to generate the turbulent driving, which does not in detail adequately represent many physical sources of energy injection. In the case of outflow-generated turbulence, considered here to be "small scale", it was indeed found that the turbulence was effectively driven on small scales. 
The close correspondence between the numerical models and the simple models of Paper I suggest also that it is not necessarily the details of the flows that are essential, but simply the range of scales on which the turbulence is present. In this sense, the modeling completed so far (Paper I and this work) adequately represent, statistically, turbulence with an outer scale that is detectable in observations. It is to be expected that more realistic driving mechanisms (e.g. as implemented by Nakamura \& Li 2007) can be investigated in future. Finally, our results recommend that simulations of randomly forced turbulence must necessarily include large-scale driving in order to replicate real molecular clouds. How this translates in detail to more realisitic driving mechanisms must be addressed in future work.

\section{Summary}

We have examined simulated observations of the density and velocity fields from numerical simulations of interstellar turbulence to investigate the scale at which energy is fed into molecular clouds. Using PCA, an observational measure of the driving scale can be obtained through the ratio of characteristic scales of the second and first eigenimages. The measured ratio of eigenimage scales, $l_{2} / l_{1}$, has the same dependence on the normalized driving scale $\left(\lambda_{\mathrm{D}} / L_{\mathrm{c}}\right)$ as derived for the normalized outer scale $\left(\lambda_{0} / L_{\mathrm{c}}\right)$ in the fBm models computed by Brunt (2003a).

Values of $l_{2} / l_{1}$ computed from spectroscopic imaging observations of molecular clouds are consistent with turbulence driven by large-scale injection of energy. We have examined a sample of 35 molecular clouds, and find that large-scale driving of turbulence provides the best match for the sample as a whole. Detailed examination of the NGC 1333 cloud shows that this cloud as a whole is best described by large-scale driving, but that the central core regions have been influenced by smallscale driving by outflows. However, while small-scale driving of turbulence through outflows can be important on small spatial scales on short time scales, it is not capable of reproducing the observed dipole structure of the second eigenimage.

The turbulence in our models was driven by random forcing, which will not represent energy injection by point-like sources very well, and future work on this issue should include more realistic methods of driving turbulence. In the meantime, we recommend that turbulence simulations that employ random forcing should ensure that the turbulence is driven on large scales to better recreate the dynamical conditions present in molecular clouds.

Acknowledgements. This work was supported by STFC Grant ST/F003277/1 to the University of Exeter, Marie Curie Re-Integration Grant MIRG-46555 (CB), and NSF grant AST 0838222 to the Five College Radio Astronomy Observatory. M-MML is supported by NSF CAREER Grant AST99-85392 and NASA Astrophysical Theory Program grant NAG5-10103. Computations analyzed here were performed at the Rechenzentrum Garching of the Max-PlanckGesellschaft. C. B. is supported by an RCUK fellowship at the University of Exeter, UK. We would like to thank Vesna Zivkov for assistance with the simulated observations, Matthew Bate and Daniel Price for helpful discussions, and the anonymous referee for a number of interesting suggestions that improved the paper.

\section{References}

Anathpindika, S., \& Whitworth, A. P. 2008, A\&A, 487, 605

Ballesteros-Paredes, J., \& Mac Low, M.-M. 2002, ApJ, 570, 734

Ballesteros-Paredes, J., Vázquez-Semadeni, E., \& Scalo, J. 1999, ApJ, 515, 286

Bally, J., Devine, D., \& Alten, V. 1996, ApJ, 668, 1028

Banerjee, R., Klessen, R. S., \& Fendt, C. 2007, ApJ, 668, 1041

Basu, S., \& Murali, C. 2001, ApJ, 551, 743

Bergin, E. A., Hartmann, L. W., Raymond, J. C., \& Ballesteros-Paredes, J. 2004, ApJ, 612, 133

Brunt, C. M. 1999, Ph.D. Thesis, University of Massachusetts, Amherst

Brunt, C. M. 2003a, ApJ, 583, 280 (Paper I)

Brunt, C. M. 2003b, ApJ, 584, 293

Brunt, C. M., \& Heyer, M. H. 2002a, ApJ, 556, 276

Brunt, C. M., \& Heyer, M. H. 2002b, ApJ, 556, 289

Brunt, C. M., \& Mac Low, M.-M. 2004, ApJ, 604, 196

Carroll, J. J., Frank, A., Blackman, E. G., Cunningham, A.J., \& Quillen, A. C. 2008 [arXiv: 0805 . 4645]

Clarke, D. 1994, NCSA Technical Report

Dale, J. E., Bonnell, I. A., Clarke, C. J., \& Bate, M. R. 2005, MNRAS, 358, 291

Dobbs, C. L., \& Bonnell, I. A. 2007, MNRAS, 374, 1115

Elmegreen, B. G. 1993, ApJ, 419, L29

Elmegreen, B. G. 2000, ApJ, 530, 277

Falgarone, É., Lis, D. C., Phillips, T. G., et al. 1994, ApJ, 436, 728

Glover, S. C. O., \& Mac Low, M.-M. 2007, ApJ, 659, 1317

Goldsmith, P. F., Heyer, M. H., Narayanan, G., et al. 2008, ApJ, 680, 428

Hartmann, L. 2003, ApJ, 585, 398

Heitsch, F., Mac Low, M.-M., \& Klessen, R. S. 2001, ApJ, 547, 280

Heyer, M. H., \& Brunt, C. M. 2004, ApJ, 615, L45

Heyer, M. H., \& Schloerb, F. P. 1997, ApJ, 475, 173

Heyer, M. H., Williams, J. P., \& Brunt, C. M. 2006, ApJ, 643, 956

Heyer, M. H., Gong, H., Ostriker, E., \& Brunt, C. M. 2008, ApJ, 680, 420

Kim, W., Ostriker, E. C., \& Stone, J. M. 2003, ApJ, 599, 1157

Klessen, R. S., Heitsch, F., \& Mac Low, M.-M. 2000, ApJ, 535, 887

Knee, L. B. G., \& Sandell, G. 2000, A\&A, 361, 671

Koda, J., Sawada, T., Hasegawa, T., \& Scoville, N. Z. 2006, ApJ, 638, 191

Larson, R. B. 1981, MNRAS, 194, 809

Lazarian, A., \& Pogosyan, D. 2000, ApJ, 537, 720

Lazarian, A., \& Pogosyan, D. 2004, ApJ, 616, 943

Mac Low, M.-M. 1999, ApJ, 524, 169

Mac Low, M.-M., \& Klessen, R. S. 2004, Rev. Mod. Phys., 76, 125

Mac Low, M.-M., Klessen, R. S., Burkert, A., \& Smith, M. D. 1998, Phys. Rev. Lett., 80, 2754

Matzner, C. 2002, ApJ, 566, 302

Miesch, M. S., \& Bally, J. 1994, ApJ, 429, 645

Nakamura, F., \& Li. Z.-Y. 2007, ApJ, 662, 395

Nakamura, F., \& Li. Z.-Y. 2008 [arXiv : 0804: 4201]

Offner, S. S. R., Klein, R. I., \& McKee, C. F. 2008, ApJ, 686, 1174

Ossenkopf, V., \& Mac Low, M.-M. 2002, A\&A, 390, 307

Ostriker, E. C., Stone, J. M., \& Gammie, C. F. 2001, ApJ, 546, 980

Pan, L., \& Padoan, P. 2009, ApJ, 692, 594

Price, D. J., \& Bate, M. R. 2008, MNRAS, 385, 1820

Quillen, A. C., Thorndike, S. L., Cunningham, A., et al. 2005, ApJ, 632, 941

Ridge, N. A., Wilson, T. L., Megeath, S. T., Allen, L. E., \& Myers, P. C. 2003, AJ, 126, 286

Ridge, N. A., Di Francesco, J., Kirk, H., et al. 2006, AJ, 131, 2921

Rosolowsky, E. W., Goodman, A. A., Wilner, D. J., \& Williams, J. 1999, ApJ, 524,887

Scalo, J. M. 1984, ApJ, 277, 556

Scalo, J. M. 1987, in Interstellar Processes, ed. D. J. Hollenbach, \& H. A. Thronson (Dordrecht: Reidel)

Schneider, N., \& Brooks, K. 2004, PASA, 21, 290

Shu, F., Adams, F. C., \& Lizano, S. 1987, ARA\&A, 25, 23

Stone, J. M., \& Norman, M. L. 1992a, ApJS, 80, 753

Stone, J. M., \& Norman, M. L. 1992b, ApJS, 80, 79

Stone, J. M., Gammie, C. F., \& Ostriker, E. C. 1998, ApJ, 508, L99

Swift, J. J., \& Welch, W. J. 2008, ApJS, 174, 202

Tamburro, D., Rix, H. W., Leroy, A. K., et al. 2009, AJ, 4424

Walawender, J., Bally, J., \& Reipurth, B. 2005, AJ, 129, 2308 